\title{
LA PERSONALIDAD COMO PREDICTORA DE DIFICULTADES COTIDIANAS EN LA ADOLESCENCIA
}

\section{PERSONALITY FEATURES AS PREDICTORS OF DIFFERENT PROBLEMS IN ADOLESCENCE}

\author{
Susana Molina Martín*, Mercedes Inda Caro**, \\ Carmen María Fernández García*** \\ Universidad de Oviedo
}

\begin{abstract}
RESUMEN
Los objetivos de este trabajo han sido: estudiar la adecuación de los datos al modelo de cinco factores e identificar rasgos de personalidad en adolescentes que manifiestan tener diversas problemáticas. Para ello se utilizaron dos instrumentos de evaluación, el Cuestionario de Personalidad para Adolescentes (16PF-APQ) y el Cuestionario Autoaplicado de Sintomas (CAS), que se administraron a ciento ocho estudiantes de último curso de educación obligatoria. Los resultados señalan que la muestra se adecua al modelo de cinco factores y que hay rasgos de personalidad que parecen explicar mejor o predecir la presencia de ciertas problemáticas en la adolescencia.
\end{abstract}

Palabras clave: Personalidad, Adolescentes, Inadaptación

\section{ABSTRACT}

The aim of this research is twofold. Firstly, it studies the adjustment of the data with the five-factor model. Secondly, it intends to describe and identify personality traits in adolescents who have manifested different problems in their daily life. For this purpose, two evaluation instruments were used: the Adolescent Personality Questionnaire (16PF-APQ) and the Adolescents Self-report of Symptoms

* Dra. Susana Molina Martín Sus campos de especialización e investigación son la educación en las ciudades, la organización y gestión de centros y las nuevas tecnologías en la educación. Correo Electrónico: smmolina@uniovi.es

** Dra. M de las Mercedes Inda Caro Actualmente se encuentra trabajando en el campo de la sociología del adolescente y la construcción social de la personalidad. Correo electrónico: indamaria@uniovi.es

*** Dra. Carmen María Fernández García Sus campos de especialización e investigación son la educación en la Unión Europea, la dimensión europea de la educación y la Educación Comparada. Correo Electrónico: fernandezcarmen@uniovi.es

Las autoras son miembros del grupo de investigación ASOCED (Análisis Sociológico y Cultural de los Procesos Escolares y Educativos). 
(C.A.S.). This last one is based on Rogers, Bagby and Dickens' instrument, Structured Interview Reported Symptoms $(1990,1991,1992)$. These tests were administered to a sample of a hundred and eight students who were in their last year of compulsory secondary education. The results of the study suggest that the sample fits with the five factor model and that there are some personality traits that seem to explain the existence of life's difficulties (questions concerning matters of anger or aggression, discouragement, worry, poor body image, alcohol or drugs, overall trouble, familiar context, scholar context and strategies coping). All the already mentioned results allow us to formulate certain indications or suggestions which would need to be taken into account in future research.

Key words: Personality, adolescents, maladjustment, overall discomfort, problems with authority, delinquency, violence, parent-child relationship, student adjustment, coping strategies.

\section{Introducción}

Identificar los atributos que describen las diferencias individuales de personalidad, ordenar sus rasgos y clasificarlos en dimensiones básicas, ha sido el objetivo de numerosos estudios. A partir de éstos, se han elaborado modelos dirigidos a explicar la personalidad, entre los que destaca en la actualidad el denominado "Modelo de Cinco Factores", y se han diseñado diversos instrumentos para su evaluación, entre los que se encuentran el NEO-PI-R de Costa y McCrae, el Cuestionario de Personalidad Zuckerman-Kuhlman (ZKPQ), el Big Five Questionnaire (BFQ) de Caprara, Barbaranelli, Borgogni y Perugini, y el Inventario de Personalidad de Cinco Factores (IP/5F) de Salgado. Además, en las últimas décadas, éstos y otros instrumentos de evaluación de la personalidad se están empleando con la intención de asociar ciertos rasgos individuales con determinadas elecciones, como la decisión profesional de ser médico (Monleón, Monleón y Rojo, 2003), y con la presencia de algunas problemáticas, como por ejemplo el consumo de heroína (Sánchez y Berjano, 1996) o el uso excesivo de Internet (Yang et al., 2005).

También ha sido estudiada la utilidad del "Modelo de Cinco Factores" para explicar los rasgos de personalidad en la infancia y la adolescencia, teniendo estos periodos vitales su propia versión de los instrumentos de evaluación. Por ejemplo, el Cuestionario de Cinco Factores para niños, BFQ-C, ha permitido explorar la personalidad en la población infantil (Carrasco, Holgado y Barrio, 2005), el NEO-PI-R y el ZKPQ-III han sido utilizados con grupos de adolescentes, observándose que se reproduce la estructura pentafactorial encontrada en muestras de adultos (Romero et al., 2002). En cuanto a la versión para adolescentes del 16PF, denominada High School Personality Questionnaire (HSPQ) de Cattell and Cattell, extrajo ocho factores para ordenar los rasgos de personalidad, que en la última edición revisada y denominada Cuestionario de Personalidad para Adolescentes, 16PF-APQ (Schuerger, 2005) pasaron a ser cinco: extraversión, independencia, autocontrol, ansiedad y dureza. Para algunos autores (Aluja y Blanch, 2002), estos cinco factores mantienen un gran nivel de concordancia en cuanto a su significado, pero no son totalmente equivalentes a los Cinco Grandes. Por ello, un primer paso en la investigación que presentamos, consiste en determinar si los datos de la muestra se ajustan a la estructura del "Modelo de Cinco Factores".

Menos estudiados han sido los rasgos de personalidad de los adolescentes en relación con algunas problemáticas que presentan en su vida cotidiana. En esta línea, el HSPQ ha sido utilizado para detectar rasgos de personalidad en aquellos adolescentes que tienen problemas variados, como la delincuencia (López y López, 2003) o el fracaso en los estudios 
(Holliday, 1996). En cuanto al 16PF-APQ, ha incorporado un inventario de síntomas, concebido como una sección que permite que el individuo señale aquellas áreas que le resultan particularmente problemáticas. Por tanto, aunque no pretende ser una medida a fondo de la personalidad clínica, es útil para detectar la existencia de una posible problemática que posteriormente deberá ser evaluada con otros instrumentos o técnicas más específicos. No obstante, parece que este tipo de investigaciones está cobrando relevancia en la actualidad, lo que contribuye a confirmar que conocer la influencia de los rasgos de personalidad sobre el desarrollo de ciertos problemas podría ser de gran utilidad para intentar prevenirlos o modificarlos, dado que como apuntan numerosos autores (Avia y Martín, 1985) en esta etapa la estructura de la personalidad se halla todavía en trance de consolidación. Parece oportuno, pues, realizar un estudio de la asociación entre las distintas variables de personalidad y algunos problemas que presentan los adolescentes, lo facilitará el desarrollo tanto de acciones preventivas como paliativas.

De modo que el presente trabajo tiene como objetivos: determinar si los datos de la muestra se ajustan a la estructura de los cinco grandes factores e indagar acerca de determinados rasgos de personalidad que guardan relación significativa con algunas dificultades que presentan los adolescentes en su vida cotidiana ${ }^{1}$.

\section{Método}

\section{Muestra}

La muestra está formada por ciento ocho adolescentes escolarizados en último curso $\left(4^{\circ}\right)$ de la Educación Secundaria Obligatoria (E.S.O.) en dos Institutos públicos de la ciudad de Oviedo. La distribución por géneros es del 52.8\% varones frente al $46.3 \%$ de mujeres, y el valor promedio en la variable edad es de $15.30\left(\mathrm{~S}_{\mathrm{x}}=0.78\right)$.

\section{Instrumentos}

Se emplean dos instrumentos de evaluación: el Cuestionario de Personalidad para Adolescentes (Schuerger, 2005) y el Cuestionario autoaplicado de síntomas, elaborado por las autoras de este artículo.

El Cuestionario de Personalidad para Adolescentes, 16PF-APQ, consiste en un autoinforme de doscientos ítems, que se encuentra estructurado en cuatro partes dirigidas a la evaluación de: la personalidad del adolescente a través de dieciséis rasgos primarios y cinco dimensiones globales, los procesos básicos de razonamiento, los intereses vocacionales y las dificultades cotidianas que pueden llegar a ser graves e incluso producir desviaciones de la conducta. Son los datos recogidos en la cuarta parte del cuestionario, bajo la denominación de "dificultades cotidianas", los que se utilizan en el presente estudio dirigido a vincular rasgos de personalidad y problemáticas que manifiestan los adolescentes.

1. Los datos utilizados en este artículo proceden del desarrollo de la investigación titulada "Evaluación de la Simulación en el contexto clínico y educativo" (UNOV -06-MB-507-1), financiada por el Vicerrectorado de Investigación y Relaciones con la Empresa de la Universidad de Oviedo. 
El Cuestionario autoaplicado de síntomas (CAS) es un instrumento en fase experimental, que se elabora a partir de una traducción del Structured Interview reported Symptoms (SIRS) de Rogers et al. (1990, 1991, 1992). Este cuestionario consta de ciento setenta y dos preguntas y permite clasificar en cuatro categorías a los sujetos en función de las puntuaciones obtenidas en las escalas primarias: honesto, indeterminado, probable fingimiento y engaño definitivo. Para el estudio que se presenta, se utilizan las puntuaciones totales del CAS con la intención de verificar la honestidad en las respuestas de los adolescentes y se analiza la contribución de algunos de sus ítems a explicar cada una de las dificultades cotidianas.

\section{Procedimiento}

En un primer momento, se contacta con los responsables del centro para explicarles el objetivo del estudio y el contenido de las pruebas. En un segundo momento se procede a la aplicación colectiva de las pruebas en cada grupo clase, en dos sesiones, de unos sesenta minutos, que transcurrieron en horario lectivo. En una primera sesión los estudiantes cumplimentaron el 16PF-APQ y en una segunda sesión realizaron el CAS, dado que se considera que los ítems son más complejos. Todas estas evaluaciones son realizadas personalmente por los miembros del equipo investigador, de modo, que se pueden contestar dudas y controlar de manera más fiable el proceso de aplicación de las pruebas.

\section{Resultados}

\section{Respecto al ajuste al modelo de cinco factores}

Un primer paso consiste en determinar si los datos de la muestra se ajustan a la estructura de cinco grandes factores. Previamente se comprueban los supuestos de normalidad y variables intercorrelacionadas. Respecto al primero, todas las escalas del 16PF-APQ tienen una distribución normal $(\mathrm{p}>.05)$, presentando una estructura de intercorrelación (K.M.O. =0.63; prueba de esfericidad de Bartlett, $\chi^{2}=369 ; p=0.000$ ). Seguidamente, se realiza un análisis factorial de componentes principales y rotación varimax. Se obtiene una solución de seis factores cuya estructura se presenta en la Tabla I. El porcentaje de varianza explicada es del $68.25 \%$.

De este primer análisis se obtienen seis factores. El primero se identifica con el factor ansiedad del 16PF-APQ, de modo que las personas con puntuaciones elevadas se caracterizan por tener dificultades para controlar sus estados emocionales y ser inseguras, impacientes, imaginativas e idealistas. El segundo se identifica con el factor autocontrol, así los sujetos con puntuaciones elevadas tienen dificultades en prestar atención a las normas, son inconformistas, flexibles en sus comportamientos y presentan dificultades en la planificación. La subdimensión dominancia puede aportar cierto grado de agresividad en personas con poco autocontrol. El factor tres alude al rasgo de introversión, indicando puntuaciones elevadas hacia sujetos solitarios, privados en sus conductas, que les cuesta abrirse a los demás, con cierta desconfianza y dificultades para mostrar sentimientos de alegría. El factor cuatro es identificado con la dimensión independencia, revelando puntuaciones altas que al indivi- 
TABLA I. Peso de cada variable en cada una de las dimensiones resultantes del análisis factorial

\begin{tabular}{|c|c|c|}
\hline Ansiedad & Autocontrol & Introversión \\
\hline $\begin{array}{l}\text { Aprensión }(0.84) \\
\text { Tensión }(0.81) \\
\text { Estabilidad }(-0.77) \\
\text { Abstracción }(0.55)\end{array}$ & $\begin{array}{l}\text { Atención a las normas }(-0.78) \\
\text { Perfeccionismo }(-0.69) \\
\text { Dominancia }(0.60)\end{array}$ & $\begin{array}{l}\text { Autosuficiencia }(0.80) \\
\text { Vigilancia }(0.64) \\
\text { Privacidad }(0.56) \\
\text { Animación }(-0.45)\end{array}$ \\
\hline Independiente & Receptiva & Racional \\
\hline $\begin{array}{l}\text { Apertura al cambio }(0.82) \\
\text { Atrevimiento }(0.76) \\
\text { Afabilidad }(0.47) \\
\text { Privacidad }(-0.35)\end{array}$ & $\begin{array}{l}\text { Sensibilidad }(0.84) \\
\text { Afabilidad }(0.63)\end{array}$ & $\begin{array}{l}\text { Razonamiento }(0.85) \\
\text { Vigilancia }(0.34)\end{array}$ \\
\hline
\end{tabular}

duo le gusta experimentar situaciones nuevas y el contacto con gente. El factor cinco viene definido por uno de los polos de la dimensión dureza, la subdimensión de personas receptivas, grupo en que se sitúan los sujetos sensibles, generosos y atentos con los demás. El factor seis, denominado racional, reúne las subdimensiones razonamiento y vigilancia, y es obtenido también por Seisdedos (Schuerger, 2005) en el análisis factorial que realiza en el proceso de estandarización de la prueba.

Para poder confirmar cuál es el número de dimensiones que mejor se ajusta al modelo, se realiza un análisis factorial confirmatorio mediante el método de máxima verosimilitud con rotación varimax con la intención de obtener factores independientes. El objetivo de este análisis factorial es confirmar que los datos se ajustan al modelo de cinco factores, aspecto que se confirma $\left(\chi^{2}=46.79 ; \mathrm{p}>0.05\right)$. Los factores obtenidos se recogen en la Tabla II. El porcentaje de varianza asociada es del $48.87 \%$. Como se puede observar, finalmente se obtienen los cinco factores recogidos también por Seisdedos (Schuerger, 2005), al ser eliminado el factor racional del proceso estadístico.

TABLA II. Peso de cada variable en cada una de las dimensiones resultantes en el análisis factorial confirmatorio

\begin{tabular}{|c|c|c|}
\hline Ansiedad & Independencia & Autocontrol \\
\hline $\begin{array}{l}\text { Aprensión }(0.81) \\
\text { Tensión }(0.80) \\
\text { Estabilidad }(-0.65) \\
\text { Abstracción }(0.46)\end{array}$ & $\begin{array}{l}\text { Atrevimiento }(0.97) \\
\text { Apertura al cambio }(0.52) \\
\text { Animación }(0.41) \\
\text { Privacidad }(-0.39) \\
\text { Atención a las normas }(-0.36)\end{array}$ & $\begin{array}{l}\text { Atención a las normas } \\
(0.72) \\
\text { Perfeccionismo }(0.54) \\
\text { Dominancia }(-0.37) \\
\text { Abstracción }(-0.35)\end{array}$ \\
\hline Extraversión & \multicolumn{2}{|c|}{ Dureza } \\
\hline Autosuficiencia (0.96) & \multicolumn{2}{|c|}{$\begin{array}{l}\text { Afabilidad }(0.86) \\
\text { Sensibilidad }(0.45) \\
\text { Apertura al cambio }(0.35)\end{array}$} \\
\hline
\end{tabular}




\section{Respecto a los rasgos de personalidad de los adolescentes que presentan dificultades}

Un segundo paso consiste en determinar cuáles son los rasgos de personalidad que predicen cada una de las dificultades cotidianas, para lo que se emplea el método de regresión múltiple con la técnica de pasos sucesivos, procedimiento que permite que cada vez que se introduce una variable en la ecuación de regresión se verifique la significatividad de las ya introducidas, eliminándose tanto las variables supresoras como las redundantes. El porcentaje de varianza explicada que se encuentra en todas las ecuaciones de regresión se sitúa entre el $19 \%$ y $45 \%$.

Seguidamente, se comentan aquellos rasgos de personalidad que aparecen asociados a las problemáticas que manifiestan los adolescentes, recogidos en la Tabla III, y agrupados en torno a cuatro ámbitos: malestar personal, meterse en problemas, dificultades en los contextos cotidianos de vida y afrontamiento deficiente.

TABLA III. Rasgos de personalidad que predicen las dificultades cotidianas del 16PF-APQ

\begin{tabular}{|l|l|r|r|r|r|r|r|}
\hline \multicolumn{1}{|c|}{$\begin{array}{c}\text { Dificultades } \\
\text { cotidianas }\end{array}$} & \multicolumn{1}{|c|}{ Variables } & Pesos & $\begin{array}{r}\text { Error } \\
\text { típico }\end{array}$ & \multicolumn{1}{c|}{ Beta } & $\mathbf{t}$ & $\mathbf{p}$ & $\mathbf{R}^{2}$ \\
\hline \multirow{4}{*}{ Desánimo } & Constante & 57.66 & 9.29 & & 6.21 & 0.000 & \\
& Estabilidad & -0.44 & 0.09 & -0.44 & -4.81 & 0.000 & 0.44 \\
& Tensión & 0.35 & 0.09 & 0.35 & 3.80 & 0.000 & \\
\hline \multirow{2}{*}{ Preocupación } & Constante & 17.95 & 4.55 & & 3.94 & 0.000 & 0.39 \\
& Ansiedad & 0.62 & 0.08 & 0.63 & 7.59 & 0.000 & \\
\hline \multirow{3}{*}{ Imagen pobre } & Constante & 61.05 & 8.87 & & 6.88 & 0.000 & \\
de sí mismo & Estabilidad & -0.44 & 0.10 & -0.43 & -4.39 & 0.000 & 0.29 \\
& Abstracción & 0.22 & 0.09 & 0.23 & 2.38 & 0.02 & \\
\hline \multirow{3}{*}{ Disconformidad } & Constante & 29.007 & 5.37 & & 5.40 & 0.000 & \\
consigo mismo & Ansiedad & 0.64 & 0.08 & 0.69 & 8.35 & 0.000 & 0.45 \\
& Autocontrol & -0.25 & 0.08 & -0.24 & -2.90 & 0.005 & \\
\hline & Constante & 21.90 & 13.56 & & 1.61 & $n .5$ & \\
& Tensión & 0.44 & 0.09 & 0.42 & 4.68 & 0.000 & \\
& Autocontrol & -0.35 & 0.10 & -0.33 & -3.44 & 0.001 & 0.36 \\
& Independencia & 0.29 & 0.10 & 0.28 & 2.85 & 0.006 & \\
& Animación & -0.23 & 0.11 & -0.22 & -2.12 & 0.04 & \\
& Autosuficiencia & 0.20 & 0.10 & 0.19 & 2.03 & 0.05 & \\
& Constante & 48.26 & 9.68 & & 4.99 & 0.000 & \\
& Tensión & 0.32 & 0.10 & 0.31 & 3.38 & 0.001 & \\
Problemas con & Razonamiento & -0.29 & 0.09 & -0.28 & -3.14 & 0.002 & 0.33 \\
la autoridad & Autocontrol & -0.28 & 0.09 & -0.27 & -3.03 & 0.003 & \\
& Vigilancia & 0.23 & 0.11 & 0.20 & 2.19 & 0.03 & \\
\hline
\end{tabular}




\begin{tabular}{|c|c|c|c|c|c|c|c|}
\hline $\begin{array}{l}\text { Dificultades } \\
\text { cotidianas }\end{array}$ & Variables & Pesos & $\begin{array}{l}\text { Error } \\
\text { típico }\end{array}$ & Beta & $\mathbf{t}$ & $p$ & $\mathbf{R}^{2}$ \\
\hline \multirow{5}{*}{$\begin{array}{l}\text { Dificultades con } \\
\text { adicciones }\end{array}$} & Constante & 6.09 & 12.19 & & 0.5 & n.s. & \multirow{5}{*}{0.19} \\
\hline & Autosuficiencia & 0.32 & 0.11 & 0.31 & 2.98 & 0.004 & \\
\hline & Apertura al cambio & 0.26 & 0.10 & 0.26 & 2.69 & 0.01 & \\
\hline & Aprensión & 0.26 & 0.09 & 0.27 & 2.80 & 0.006 & \\
\hline & Animación & 0.22 & 0.11 & 0.21 & 2.01 & 0.05 & \\
\hline \multirow{4}{*}{$\begin{array}{l}\text { Dificultades en } \\
\text { casa }\end{array}$} & Constante & 68.77 & 10.04 & & 6.85 & 0.000 & \multirow{4}{*}{0.45} \\
\hline & Estabilidad & -0.35 & 0.10 & -0.34 & -3.67 & 0.000 & \\
\hline & Razonamiento & -0.35 & 0.08 & -0.34 & -4.24 & 0.000 & \\
\hline & Tensión & 0.34 & 0.09 & 0.33 & 3.63 & 0.000 & \\
\hline \multirow{5}{*}{$\begin{array}{l}\text { Dificultades en } \\
\text { el colegio }\end{array}$} & Constante & 41.55 & 10.42 & & 3.99 & 0.000 & \multirow{5}{*}{0.30} \\
\hline & Abstracción & 0.26 & 0.09 & 0.28 & 2.97 & 0.004 & \\
\hline & Razonamiento & -0.26 & 0.09 & -0.26 & -2.89 & 0.005 & \\
\hline & Vigilancia & 0.25 & 0.10 & 0.23 & 2.48 & 0.01 & \\
\hline & Perfeccionismo & -0.21 & 0.10 & -0.21 & -2.24 & 0.03 & \\
\hline \multirow{4}{*}{$\begin{array}{l}\text { Afrontamiento } \\
\text { deficiente }\end{array}$} & Constante & 50.12 & 8.57 & & 5.85 & 0.000 & \multirow{4}{*}{0.28} \\
\hline & Tensión & 0.44 & 0.09 & 0.42 & 4.65 & 0.000 & \\
\hline & Razonamiento & -0.28 & 0.09 & -0.28 & -3 & 0.004 & \\
\hline & Afabilidad & -0.21 & 0.09 & -0.21 & -2.35 & 0.02 & \\
\hline
\end{tabular}

\section{Adolescentes con problemas de malestar personal}

Los problemas de malestar personal (desánimo, preocupación e imagen pobre de sí mismo), que numerosas investigaciones sitúan entre los trastornos más frecuentes de la esfera emocional en adolescentes (Hernández y Rodrigo, 2003), aparecen asociados a una combinación de bajo autocontrol y elevada ansiedad.

Concretamente, en adolescentes que muestran tendencia al desánimo (ánimo bajo, apatía, alienación, depresión, sentimientos suicidas) se observan puntuaciones bajas en estabilidad y autocontrol, junto a puntuaciones elevadas en ansiedad y tensión. Es decir, ser trata de individuos que pueden experimentar sentimientos de cierta falta de control sobre su vida y responden reaccionando contra ella, en vez de adaptarse a las alternativas que se le presentan. Si el desánimo aparece acompañado de un déficit en las estrategias de afrontamiento, podría ser un indicador de la probabilidad de suicidio (Schuerger, 2005, 101).

En el caso de aquellos adolescentes que manifiestan sentirse preocupados (miedos, ... se observan puntuaciones elevadas en ansiedad, que puede producirse ante sucesos generados externamente o por ellos mismos y que consideran una amenaza, sea ésta real o percibida. Si el sujeto además tiene dificultades para controlar sus emociones o reacciones, podría actuar con conductas contraproducentes.

Cuando los adolescentes afirman que tienen una imagen pobre de sí mismos, obtienen una puntuación baja en estabilidad emocional y elevada en ansiedad y abstracción. Es decir, 
podría tratarse de un individuo que se siente incapaz de controlar su vida, que no se adapta al contexto y sus demandas, pudiendo prestar más atención a sus pensamientos que a las cosas que le rodean. Habitualmente tener una imagen pobre de uno mismo se asocia con preocupaciones referidas al propio cuerpo, trastornos de alimentación (Alemán et al, 2001; León, Sepúlveda y Botella, 2001), al sobrepeso y la apariencia en general.

\section{Adolescentes que se meten en problemas de adicción, agresión o con la autoridad}

Específicamente, apertura al cambio, aprensión, animación y autosuficiencia, son algunos rasgos de la personalidad de aquellos adolescentes que manifiestan problemas con adicciones, variando su incidencia en función del tipo de sustancia. Se trata de individuos que precisan una estimulación intensa para no aburrirse (mucha actividad y excitación), lo que les lleva a experimentar nuevas prácticas o a realizar actividades arriesgadas (como el consumo de drogas y alcohol). Podrían ser impulsivos en sus acciones, poco sociables, tomar decisiones en solitario y por su cuenta sin pedir ayuda aunque la necesiten, e incluso ser aprensivos e inseguros, lo que contribuye a que se sientan heridos si los demás no les aceptan. Llorens, Palmer y Perelló (2005) coinciden en situar como factores de riesgo: la impulsividad, la conducta antisocial, la búsqueda de sensaciones mediante experiencias variadas y nuevas (aceptando los riesgos físicos y sociales) y el pobre concepto de sí mismos. Además, se ha encontrado que hay más mujeres que dicen tener adicciones que varones $(\mathrm{p}=0.01)$, siendo el valor promedio en esta variable superior (70.89 \& 56.40). Podría barajarse como hipótesis que las diferencias encontradas se deben a una falta de sinceridad por parte de los chicos. Para comprobarlo, se compararon ambos grupos en la variable manipulación de la imagen, pero no se encontraron diferencias estadísticamente significativas, e incluso los varones obtuvieron una media inferior a las mujeres (47.43 vs 53.31), con lo cual se tiene que desechar la hipótesis. En este sentido, algunos estudios han verificado la existencia de diferencias en la adicción a ciertas sustancias en función del género, siendo una de ellas el tabaco (Llorens, Palmer y Perelló, 2005).

Los rasgos de personalidad, en el caso de los adolescentes que presentan problemas con la autoridad (robos, etc.), son: vigilancia, bajo autocontrol y razonamiento, junto puntuaciones elevadas en tensión. Lo que indica que podría tratarse de individuos que no se creen bien comprendidos, que piensan que los demás se pueden aprovechar de ellos, lo que les hace desconfiados y les lleva a estar en guardia. Esta postura vigilante, provoca una mayor tensión nerviosa, lo que a veces puede perturbarles e impedir el razonamiento y una acción efectiva. Además, pueden tener dificultades para controlar sus reacciones, no siendo capaces de prevenir el desbordamiento o descontrol, lo que podría llevarles a actuar con conductas contraproducentes. Los resultados obtenidos son parcialmente coincidentes con el patrón caracterizado por impulsividad, bajo autocontrol, poca interiorización y respeto a las normas, ansiedad, extraversión e independencia, presentado por López y López (2003).

En cuanto a los adolescentes que tienen problemas de agresión, es decir, que manifiestan acciones o sentimientos violentos hacia otras personas, puntúan alto en independencia y autosuficiencia, mientras que puntúan bajo en autocontrol y animación. Podría tratarse de un sujeto activo y enérgicamente determinado en los pensamientos y acciones, que tiende a no acomodarse a su ambiente, lo que le lleva a intentar cosas nuevas y a retar lo estableci- 
do. Pero al no estar su conducta atemperada, podrían aparecer efectos desagradables. Es decir, podría actuar de forma desinhibida y atendiendo a sus propias urgencias, prefiriendo tomar las decisiones por su cuenta, pasando por alto los aspectos interpersonales y las consecuencias de sus acciones. No obstante, bajo este perfil, también cabe alguien callado o apagado, que inhibe su espontaneidad.

\section{Adolescentes con problemas en casa o en el colegio}

Concretamente, aquellos adolescentes que manifiestan tener dificultades en su casa puntúan bajo en estabilidad emocional, lo que les lleva a experimentar una cierta falta de control sobre su vida. Además, se perciben incapaces de adaptarse a la vida familiar y a sus demandas, lo que provoca ansiedad. Esto, junto a puntuaciones altas en tensión nerviosa, puede perturbarles e impedir el razonamiento y una acción efectiva.

En cuanto a los adolescentes que afirman tener dificultades en el colegio, presentan puntuaciones altas en abstracción y vigilancia, a la vez que son poco perfeccionistas y tienen un razonamiento bajo. Es decir, se trata de un individuo que puede creerse incomprendido, que deja las cosas a su suerte, y que los demás pueden considerar perezoso, desorganizado y con falta de preparación. Puede llegar a no tener una clara motivación para comportarse de una manera planificada y organizada si no percibe la conducta como importante. Además, dirige su atención y pensamiento más hacia otros temas que hacia lo que tiene que hacer. Puede estar ocupado pensando, imaginando, fantaseando, ensimismado en sus pensamientos, poniendo más atención a éstos que a las cosas que le rodean, lo que podría llevarle a un mayor número de respuestas incorrectas. Diversos estudios (Fierro, 2002; Pelechano y González, 2004) coinciden en señalar que el éxito académico no depende tanto de la capacidad intelectual, como de la extraversión social y la exigencia ante el estudio.

\section{Adolescentes con problemas de afrontamiento}

Las estrategias de afrontamiento son necesarias para los problemas educativos, afectivos, personales y familiares que el adolescente pueda tener, dado que tienen que ver con la competencia social, la eficacia en las tareas, con los diferentes estilos o métodos para resolver problemas y con una actitud y valores razonables. De acuerdo con los resultados obtenidos, los adolescentes que manifiestan un afrontamiento deficiente se caracterizan por ser poco afables, tener un razonamiento bajo y encontrarse en tensión. Podría tratarse de un sujeto reservado y cauto en sus implicaciones y contactos (que reserva el problema para sí), ansioso, preocupado por el problema, con elevada tensión nerviosa que le puede llevar a la distracción y perturbarle, impidiendo una acción efectiva. Diversas investigaciones apoyan estos resultados (Fatin, Florentino, Correché, 2005; González et al., 2002), al considerar que unas estrategias de afrontamiento adecuadas, como son la preocupación productiva, la búsqueda de apoyo social (amigos, profesionales, etc.) que implica manejo directo del problema a través de la orientación que otros puedan proveer, etc., correlacionan con estilos de personalidad sociables.

Para completar este bloque de análisis, la muestra ha sido evaluada también mediante el CAS. Concretamente, se quiso determinar cuáles eran los ítems del cuestionario que mejor 
contribuían a definir cada concepto de dificultades cotidianas descritas por el 16PF-APQ. En la Tabla IV, se presenta el coeficiente de determinación para cada una de las variables y el peso de los ítems más destacados en la definición de los problemas que puede tener un adolescente en su vida diaria. En un análisis cualitativo, se observan relaciones conceptuales entre ambos componentes, que guardan relación con las dimensiones de personalidad que recoge la Tabla III, respecto a cuáles van a pronosticar una dificultad.

TABLA IV. Relaciones entre los ítems del CAS y del 16PF-APQ

\begin{tabular}{|c|c|c|c|}
\hline $\begin{array}{l}\text { Dificultades } \\
\text { cotidianas }\end{array}$ & \multicolumn{3}{|c|}{ Ítem del CAS } \\
\hline \multirow{6}{*}{$\begin{array}{l}\text { Desánimo } \\
\left(R^{2}=0.56\right)\end{array}$} & Ítem 30 & $\begin{array}{l}\text { ¿A veces, tienes problemas de concentración cuando } \\
\text { estás disgustado? }\end{array}$ & 0.27 \\
\hline & Ítem 127 & $\begin{array}{l}\text { ¿Te sientes más confundido o alterado al mediodía que } \\
\text { en otros momentos? }\end{array}$ & -0.49 \\
\hline & Ítem 25 & $\begin{array}{l}\text { A veces cuando te encuentras sin ánimos, sin fuerzas, } \\
\text { sin ganas de hacer nada, ¿te sientes aturdido o como } \\
\text { si te desmayaras? }\end{array}$ & 0.44 \\
\hline & Ítem 37 & $\begin{array}{l}\text { ¿Tienes una idea clara acerca de lo que es moralmente } \\
\text { correcto e incorrecto? }\end{array}$ & -0.15 \\
\hline & Ítem 66 & $\begin{array}{l}\text { ¿Tienes dificultades para recordar el nombre de las } \\
\text { personas que acabas de conocer? }\end{array}$ & 0.29 \\
\hline & Ítem 145 & $\begin{array}{l}\text { ¿Tiendes a suspirar cuando hablas con otras } \\
\text { personas? }\end{array}$ & 0.21 \\
\hline \multirow{7}{*}{$\begin{array}{l}\text { Preocupación } \\
\left(R^{2}=0.67\right)\end{array}$} & Ítem 37 & $\begin{array}{l}\text { ¿Tienes una idea clara acerca de lo que es moralmente } \\
\text { correcto e incorrecto? }\end{array}$ & -0.48 \\
\hline & Ítem 162 & $\begin{array}{l}\text { ¿Tiene dificultades importantes con personas que te } \\
\text { hagan enfadar? }\end{array}$ & 0.33 \\
\hline & Ítem 66 & $\begin{array}{l}\text { ¿Tienes dificultades para recordar el nombre de las } \\
\text { personas que acabas de conocer? }\end{array}$ & 0.46 \\
\hline & Ítem 118 & $\begin{array}{l}\text { ¿Te has levantado alguna vez por la mañana y te has } \\
\text { dado cuenta de que todos tus problemas han } \\
\text { desaparecido... sintiéndote completamente bien? }\end{array}$ & -0.22 \\
\hline & Ítem 133 & $\begin{array}{l}\text { ¿Cuándo hablas con otros, cuchicheas o susurras sin } \\
\text { razón aparente? }\end{array}$ & 0.26 \\
\hline & Ítem 26 & ¿Tienes algunos secretos que nadie conoce? & 0.20 \\
\hline & Ítem 44 & $\begin{array}{l}\text { ¿Te gusta a veces engañar o llevar a error al orientador } \\
\text { o a los profesores? }\end{array}$ & 0.15 \\
\hline
\end{tabular}




\begin{tabular}{|c|c|c|c|}
\hline $\begin{array}{l}\text { Dificultades } \\
\text { cotidianas }\end{array}$ & \multicolumn{3}{|c|}{ Ítem del CAS } \\
\hline \multirow{6}{*}{$\begin{array}{l}\text { Imagen pobre } \\
\text { de sí mismo } \\
\left(R^{2}=0.40\right)\end{array}$} & Ítem 18 & $\begin{array}{l}\text { ¿Tus pensamientos son dados a conocer a mucha } \\
\text { gente, de tal manera que ellos saben lo que estás } \\
\text { pensando? }\end{array}$ & 0.30 \\
\hline & Ítem 66 & $\begin{array}{l}\text { ¿Tienes dificultades para recordar el nombre de las } \\
\text { personas que acabas de conocer? }\end{array}$ & 0.24 \\
\hline & Ítem 112 & ¿Te enfadas cuando otros intentan aprovecharse de ti? & -0.34 \\
\hline & Ítem 56 & $\begin{array}{l}\text { ¿Cuándo estás nervioso, sueles reírte de cosas que no } \\
\text { son graciosas? }\end{array}$ & 0.27 \\
\hline & Ítem 162 & $\begin{array}{l}\text { ¿Tiene dificultades importantes con personas que te } \\
\text { hagan enfadar? }\end{array}$ & 0.39 \\
\hline & İ́m 157 & $\begin{array}{l}\text { ¿Has tenido algún problema importante con tu } \\
\text { concentración? }\end{array}$ & -0.37 \\
\hline \multirow{3}{*}{$\begin{array}{l}\text { Ira-Agresión } \\
\left(R^{2}=0.24\right)\end{array}$} & Ítem 127 & $\begin{array}{l}\text { ¿Te sientes más confundido o alterado al mediodía que } \\
\text { en otros momentos? }\end{array}$ & -0.39 \\
\hline & Ítem 45 & $\begin{array}{l}\text { ¿Te apetecería a veces insultar a las personas que han } \\
\text { sido mal educadas contigo? }\end{array}$ & 0.38 \\
\hline & Ítem 147 & $\begin{array}{l}\text { ¿Has tenido alguna vez pensamientos de "ya me las } \\
\text { pagará", cuando alguien hiere tus sentimientos? }\end{array}$ & -0.30 \\
\hline \multirow{3}{*}{$\begin{array}{l}\text { Problemas con } \\
\text { la autoridad } \\
\left(R^{2}=0.30\right)\end{array}$} & Ítem 127 & $\begin{array}{l}\text { ¿Te sientes más confundido o alterado al mediodía que } \\
\text { en otros momentos? }\end{array}$ & -0.38 \\
\hline & Ítem 38 & $\begin{array}{l}\text { En épocas en las que estás más activo físicamente, } \\
\text { ¿tienes miedo que alguien pueda hacerte daño? }\end{array}$ & -0.21 \\
\hline & Ítem 25 & $\begin{array}{l}\text { A veces cuando te encuentras sin ánimos, sin fuerzas, } \\
\text { sin ganas de hacer nada, ¿te sientes aturdido o como } \\
\text { si te desmayaras? }\end{array}$ & 0.23 \\
\hline \multirow{3}{*}{$\begin{array}{l}\text { Dificultades con } \\
\text { las adicciones } \\
\left(\mathrm{R}^{2}=0.31\right)\end{array}$} & Ítem 127 & $\begin{array}{l}\text { ¿Te sientes más confundido o alterado al mediodía que } \\
\text { en otros momentos? }\end{array}$ & -0.34 \\
\hline & Ítem 38 & $\begin{array}{l}\text { En épocas en las que estás más activo físicamente, } \\
\text { ¿tienes miedo que alguien pueda hacerte daño? }\end{array}$ & -0.25 \\
\hline & Ítem 23 & $\begin{array}{l}\text { ¿Aparecen la mayoría de tus problemas } \\
\text { repentinamente? }\end{array}$ & 0.35 \\
\hline
\end{tabular}




\begin{tabular}{|c|c|c|c|}
\hline $\begin{array}{l}\text { Dificultades } \\
\text { cotidianas }\end{array}$ & \multicolumn{3}{|c|}{ Ítem del CAS } \\
\hline \multirow{5}{*}{$\begin{array}{l}\text { Dificultades en } \\
\text { casa }\left(R^{2}=0.35\right)\end{array}$} & Ítem 107 & $\begin{array}{l}\text { ¿Deseas a veces la paz en el mundo y el fin de la } \\
\text { pobreza? }\end{array}$ & -0.38 \\
\hline & Ítem 168 & $\begin{array}{l}\text { ¿Tienes problemas graves con reaccionar de manera } \\
\text { exagerada ante situaciones difíciles? }\end{array}$ & 0.48 \\
\hline & Ítem 158 & $\begin{array}{l}\text { ¿Has tenido algún problema importante con } \\
\text { pensamientos perturbadores o extraños? }\end{array}$ & -0.40 \\
\hline & Ítem 127 & $\begin{array}{l}\text { ¿Te sientes más confundido o alterado al mediodía que } \\
\text { en otros momentos? }\end{array}$ & -0.24 \\
\hline & Ítem 61 & ¿Tienes miedo de hacerte mayor? & 0.21 \\
\hline $\begin{array}{l}\text { Dificultades en } \\
\text { el colegio } \\
\left(R^{2}=0.26\right)\end{array}$ & Ítem 165 & ¿Tienes problemas graves con sentirte muy deprimido? & 0.26 \\
\hline \multirow{5}{*}{$\begin{array}{l}\text { Afrontamiento } \\
\text { deficiente } \\
\left(R^{2}=0.38\right)\end{array}$} & Ítem 108 & $\begin{array}{l}\text { ¿Has podido solucionar con éxito y sin ayuda tus } \\
\text { problemas emocionales? }\end{array}$ & -0.41 \\
\hline & Ítem 5 & $\begin{array}{l}\text { ¿Tienes dificultades serias con tener dudas sobre ti } \\
\text { mismo? }\end{array}$ & -0.37 \\
\hline & Ítem 45 & $\begin{array}{l}\text { ¿Te apetecería a veces insultar a las personas que han } \\
\text { sido mal educadas contigo? }\end{array}$ & 0.35 \\
\hline & Ítem 123 & $\begin{array}{l}\text { ¿Has pasado alguna vez temporadas preocupado por } \\
\text { tu futuro? }\end{array}$ & -0.35 \\
\hline & Ítem 109 & $\begin{array}{l}\text { ¿Tus problemas emocionales surgen de repente, de } \\
\text { modo que un día te sientes completamente normal y al } \\
\text { día siguiente estás muy preocupado? }\end{array}$ & 0.28 \\
\hline
\end{tabular}

\section{Conclusiones}

Este estudio permite confirmar la presencia de rasgos específicos de personalidad en adolescentes que señalan en el 16PF-APQ una posible problemática en alguna de las cuatro áreas: malestar personal, meterse en problemas, dificultades en la familia o en el colegio y afrontamiento deficiente. Los rasgos de personalidad aportan una información fundamental en la que se debe profundizar desde el ámbito del diagnóstico. Cada caso requiere un estudio individual y pormenorizado, donde además se analicen las causas sociales, tanto a nivel familiar, de grupo de iguales, como del contexto escolar, que pueden estar influyendo en el desarrollo de la conducta no deseada.

Conocer la influencia de los rasgos de personalidad sobre el desarrollo de ciertos problemas podría ser de gran utilidad para aquellos profesionales que trabajan con adolescentes, como son los orientadores de los institutos. Si se entiende que los rasgos de personalidad son predisposiciones estables a comportarse y reaccionar emocionalmente de una determinada manera o según un patrón característico (López y López, 2003), que están tanto en su 
génesis como en su mantenimiento influidos por el ambiente y que en la etapa de la adolescencia la estructura de la personalidad se halla todavía en trance de consolidación, entonces el conocer aquellos aspectos que inciden sobre ellos podría ser de gran utilidad para desarrollar procedimientos de prevención o efectivizar la ayuda en caso de que sea necesario. Se trataría, pues, de una actuación en un momento fundamental en la configuración de la personalidad, y, que además, podría prevenir la presencia de algunas problemáticas en la edad adulta.

Para finalizar, cabe decir que las autoras son conscientes de las limitaciones de este trabajo. Respecto a los aspectos metodológicos, hay que señalar que la muestra en ningún caso se puede considerar representativa de la población adolescente, siendo preciso ampliarla en investigaciones posteriores. Se trata de un estudio inicial cuyos datos no son todavía generalizables, aunque cuenta con dos aspectos positivos. En primer lugar, los resultados parecen coincidir con los de otras investigaciones realizadas acerca de cada una de las temáticas trabajadas. En segundo lugar, cabe tener presente que el que cada estudiante tenga que cumplimentar dos cuestionarios ha permitido la verificación de los datos, primero, al poner en relación los ítems de ambos cuestionarios relativos a los diversos rasgos de la personalidad con las problemáticas que presentan los adolescentes, $\mathrm{y}$, segundo, al detectar mediante el CAS la sinceridad del sujeto. Sin embargo, se necesitaran futuros estudios que afiancen los resultados obtenidos en este trabajo tanto a nivel conceptual como estadístico.

\section{Referencias bibliográficas}

Aleman, A. et al. (2001). La imagen corporal en los trastornos alimentarios. Psicothema, 13 (4), 533 538.

Aluja, A. y Blanch, A. (2002). Relación entre la estructura del 16PF-5 y el modelo de cinco grandes factores de personalidad. Boletín de Psicología, 75, 7-18.

Avia, M. D. y Martín, J. (1985). Cambio y continuidad en la personalidad. En M. Carretero et al (Comp.), Psicología evolutiva: adolescencia, madurez y senectud (pp. 177-200). Madrid: Alianza.

Carrasco, M. A.; Holgado, F. P. y Barrio, M. V. (2005). Dimensionalidad del cuestionario de los cinco grandes (BFQ-N) en población infantil española. Psicothema, 17 (2), 286-291.

Costa, P. T., y McCrae, R. R. (1985). Tne NEO personality Inventory manual. Odessa, Florida: Psychological Assessment Resources.

Digman, J. M. (1990). Personality Structure: Emergence of the five-factor model. Annual Review Of Psychology, 41, 417-440.

Fatin, M. B. (2006). Perfil de personalidad y consumo de drogas en adolescentes escolarizados. Adicciones, Vol. 18 (3), 285-292.

Fierro, C. (2002). Patrón de rasgos personales y comportamiento escolar en jóvenes. Revista de Educación, 329, 373-392.

González, R. et al. (2002). Relación entre estilos y estrategias de afrontamiento y bienestar psicológico en adolescentes. Psicothema, 14 (2), 363-368.

Hernandez, R. y Rodrigo, M. (2003). Ansiedad, depresión y conducta suicida en la adolescencia. Medicine, 116, 6199-6208.

Holliday, G. A. (1996). Personality Attributes of High IQ/High Achieving Gifted adolescents: implications of the personal styles model. Journal for the Education of the Gifted, 20 (1), 84-102. 
León, J. A., Sepúlveda, A. R. y Botella, J. (2001). La alteración de la imagen corporal en los trastornos de la alimentación: un meta-análisis. Psicothema, 13 (1), 7-16.

Llorens, N., Palmer, A. y Perelló, M. J. (2005). Características de personalidad en adolescentes como predictores de la conducta de consumo de sustancias psicoactivas. Trastornos Adictivos: Órgano Oficial de la Sociedad Española de Toxicomanias, 7 (2), 44-50.

López, C. y López, J. R. (2003). Rasgos de personalidad y conducta antisocial delictiva. Psicopatología Clínica Legal y Forense, 3 (2), 5-19.

Monleón, A. M., Monleón, P. J. y Rojo, J. (2003) Factores de personalidad y predilección por las distintas especialidades en los estudiantes de medicina. Anales de Psiquiatría, 19 (8), 337-342

Norman, W. T. (1963). Toward an adequate taxonomy of personlaity attributes: Replicated factor structure inpeer nomination personality ratings. Journal of Abnormal and Social Psychology, 66, 574-583.

Pelechano, V. y González, P. (2004). Personalidad, conocimiento relacionado con la sabiduría y rendimiento académico en adolescentes. Análisis y Modificación de Conducta, 30 (134), 977-990.

Rogers, R. et al. (1990). Cross validation of the SIRS with a correctional sample. Behavioral Sciences and the Law, 8, 85-92.

Rogers, R. et al. (1991). Standardized assessment of malingering: Validation of the SIRS. Psychological Assessment, 3, 89-96.

Rogers, R. et al. (1992). Structured Interview of Reported Symptoms (SIRS). Florida: PAR: Psychological Assessment Resource, Inc.

Romero, E. et al. (2002). La estructura de los rasgos de personalidad en adolescentes: el Modelo de Cinco Factores y los cinco alternativos. Psicothema, 14 (1), 134-143.

Sánchez, E. y Berjano, E. (1996). Características de personalidad en sujetos drogodependientes. Psicothema, 8 (3), 457-463.

Schuerger, J. M. (2005). 16PF-APQ. Cuestionario de Personalidad para Adolescentes. Manual (N. Seisdedos, Trans.). Madrid: TEA Ediciones.

Yang, CK. et al (2005). SCL-90-R and 16 PF profiles of senior high school students with excessive Internet use. Canadian Journal of Psychiatry-Revue Canadienne de Psychiatre, 50, 407-414.

Fecha de recepción: 16-04-2007

Fecha de revisión: 05-10-2008

Fecha de aceptación: 05-05-2009 